OPEN ACCESS

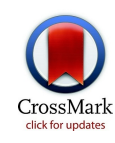

For numbered affiliations see end of article.

Correspondence to:

Q Shen qing.shen@ki.se

Additional material is published

online only. To view please visit

the journal online.

Cite this as: $B M / 2016 ; 354: i 4218$

http://dx.doi.org/10.1136/bmj.i4218

Accepted: 18 July 2016

\section{Injuries before and after diagnosis of cancer: nationwide register based study}

\author{
Qing Shen, ${ }^{1}$ Donghao Lu, ${ }^{1}$ Maria E C Schelin, ${ }^{2,3}$ Anna Jöud, ${ }^{2,4}$ Yang Cao,, ${ }^{5,6}$ Hans-Olov Adami, ${ }^{1,7,8}$ \\ Sven Cnattingius, ${ }^{9}$ Katja Fall, ${ }^{6}$ Unnur Valdimarsdóttir, ${ }^{1,8,10}$ Fang Fang ${ }^{1}$
}

\author{
ABSTRACT \\ OBJECTIVE \\ To examine the relative risks of iatrogenic and \\ non-iatrogenic injuries during the period shortly before \\ and after a diagnosis of cancer.
}

DESIGN

Nationwide register based study.

\section{SETTING}

Swedish national population and health registers.

\section{PARTICIPANTS}

720901 patients with diagnosis of cancer, 1991-2009, in Sweden.

\section{MAIN OUTCOME MEASURES}

All hospital admissions in patients with cancer with a main discharge diagnosis of iatrogenic (from medical complications) or non-iatrogenic injuries in 1990-2010 identified from the Swedish patient register. Conditional Poisson regression was used to compare the incidence rate of injuries during the "diagnostic period" (16 weeks before to 16 weeks after diagnosis) with the incidence rate during a "pre-diagnostic period" (the same 32 weeks one year before diagnosis) among the same patients.

\section{RESULTS}

During the diagnostic period, there were 7306 iatrogenic (incidence rate 0.60 per 1000 person months) and 8331 non-iatrogenic injuries (incidence rate 0.69 per 1000 person months). For iatrogenic injuries, the incidence rate ratio was 7.0 (95\% confidence interval 6.6 to 7.4 ) during the diagnostic period compared with the pre-diagnostic period. The increase in risk started two weeks before cancer diagnosis and peaked during the two weeks after diagnosis (48.6, 37.3 to 63.5). For non-iatrogenic injuries, the incidence rate ratio was 1.9 (1.8 to 2.0) during the diagnostic period compared with the

\section{WHAT IS ALREADY KNOWN ON THE TOPIC}

Complications from medical procedures and drug treatment are common among patients with cancer

Increased risks of suicide and accidental death after diagnosis have been reported The diagnostic process of cancer is highly stressful

\section{WHAT THIS STUDY ADDS}

This is the first study to systemically characterise and quantify the increased risk of iatrogenic and non-iatrogenic injuries during the process of cancer diagnosis

Among non-iatrogenic injuries, there are similarly increased risks of unintentional injuries before and after diagnosis, whereas the increased risk of intentional injuries was most prominent after diagnosis

Different mechanisms could underlie the increased risks of intentional and unintentional injuries around diagnosis pre-diagnostic period. The increase in risk began four weeks before diagnosis and peaked during the two weeks before diagnosis $(5.3,4.6$ to 6.1$)$. There were increased risks of both types of injury during the diagnostic period for all common cancers, with the smallest risk increase noted for non-melanoma skin cancer.

\section{CONCLUSIONS}

Patients with cancer have highly increased risks of both iatrogenic and non-iatrogenic injuries requiring inpatient care shortly before and after their diagnosis. These findings shed further light on the total burden of medical complications and call for prevention of intentional and unintentional injuries during the diagnostic process of cancer.

\section{Introduction}

In patients with cancer, injury is one of the leading causes of non-cancer mortality. ${ }^{1}$ Iatrogenic injuries (that is, complications from medical procedures and drug treatment) are common in those with cancer ${ }^{23}$ and have been shown to increase mortality among patients with colorectal, prostate, and breast cancers. ${ }^{4-7}$ Risk of non-iatrogenic injuries (that is, injuries not resulting from medical interventions or patients' health seeking behaviours-a sequence of remedial actions that individuals undertake to rectify perceived ill health) is also increasingly recognised among patients with cancer. Increased risks of self injury, mainly suicide, ${ }^{89}$ and accidental death, especially during the first year after diagnosis, ${ }^{1011}$ have been reported among people with cancer. The vulnerability to suicide and accidental death might be triggered by both the symptoms of the disease and the overwhelming psychological stress people can experience when they receive a diagnosis of cancer. ${ }^{912}$

Previous studies have predominantly examined the risk of injuries, mostly fatal injuries, either after diagnosis of cancer ${ }^{11}$ or after treatment for cancer. It is, however, unknown whether the risk of injuries is also increased during the time period before the diagnosis. Evidence suggests that the diagnostic process of cancer entails severe emotional distress, regardless of the eventual result, ${ }^{13-17}$ and could therefore increase the risk of non-iatrogenic injuries (intentional or unintentional). To this end, we analysed the risks of both iatrogenic and non-iatrogenic injuries during the weeks before and after diagnosis using a nationwide study sample in Sweden.

\section{Methods}

Study design

The Swedish cancer register collects information on all newly diagnosed cases of cancer in the entire country 
since 1958 and the coverage approaches 100\%. ${ }^{18}$ Based on this register, we included 740114 unique patients with a first cancer diagnosed during 1991-2009. Cases diagnosed through autopsy were not included. The median age at diagnosis was 69 , and $51.7 \%$ of the patients were male.

We used the Swedish revision of ICD-7 (international classification of diseases, seventh revision) to classify cancers as prostate cancer $(n=123837)$, breast cancer ( $n=101458$; women only), colorectal cancer ( $n=84527)$, non-melanoma skin cancer $(n=33409)$, lymphatic or hematopoietic cancers $(n=52266)$, lung cancer $(n=49491)$, cancers of the central nervous system $(n=21199)$, cancers with an expectedly short survival $(n=34627$; "severe cancers" including cancers in the oesophagus, liver, and pancreas), and other cancers $(\mathrm{n}=220087)$. Lifestyle factors, such as smoking and drinking alcohol, might modify the association between diagnosis and injuries. ${ }^{1920}$ We therefore separately analysed smoking related cancers (cancers of mouth, nasopharynx, oesophagus, pancreas, lung, kidney, bladder, and urinary tract; $\mathrm{n}=116501$ ) and alcohol related cancers (cancers of mouth, larynx, oesophagus, biliary duct, and liver; $\mathrm{n}=29511$ ). ICD-7 codes used for different classifications are listed in section 1 of the appendix.

Cancer and injuries can share risk factors, leading to spurious associations between these conditions. To eliminate such confounding, we used comparisons within individuals, in which we compared the risk of injuries during the diagnostic period with the risk during a reference period, in the same patients.

\section{Definitions of time periods}

The Swedish cancer register provides little information regarding the course of the diagnostic process for cancer, other than the date of diagnosis. In Sweden, the median waiting time, defined as the time between the first referral to a specialist for suspected cancer and the primary cancer treatment, is around two months. ${ }^{21}$ Because symptoms of cancer, as well as the clinical evaluation of a potential cancer, probably start much earlier than the first specialist visit for most patients and the waiting time varies largely for different cancers, we defined the diagnostic period as the 16 weeks before until the 16 weeks after the date of diagnosis. Accordingly, we defined the same 32 week period one year before diagnosis as the reference period ("pre-diagnostic period"). To examine the increase in risk of injuries during the diagnostic period compared with later on, we further defined the same 32 weeks one year after diagnosis as the post-diagnostic period. Among the 740114 patients with cancer, 13 died before the diagnostic period and were excluded from all analyses. Patients who died before the post-diagnostic period contributed only to the analyses of the diagnostic period ( $\mathrm{n}=138947)$.

\section{Ascertainment of injuries}

Using the individually unique national registration numbers assigned to all Swedish residents, we cross linked patients identified in the cancer register to the Swedish patient register. The patient register compiles hospital discharge records from 1964-65 onward and is nationwide from 1987 onward. The register includes information on dates of admission and discharge, as well as the primary and up to 28 secondary discharge diagnoses. ${ }^{22}$ We identified all hospital admissions of the participating patients with cancer during 1990-2010. Individuals with incomplete information about any of the admissions during this period, including dates of admission and discharge or main discharge diagnosis, were excluded from the analyses ( $n=19200)$, leaving 720901 (97.4\%) in the final analyses. We ascertained all admissions of these patients due to injuries during the pre-diagnostic, diagnostic, and post-diagnostic periods, using ICD-9 codes 800-999 for main discharge diagnosis and E807-E999 for external causes (1990-96) as well as ICD-10 codes S00-T98 for main discharge diagnosis and V01-Y98 for external causes (1997-2010). Consecutive hospital discharges that were within a week of each other and had the same injury diagnosis were considered as one injury event.

Injuries associated with a healthcare related diagnosis, treatment, or other medical complications were referred as iatrogenic injuries. Injuries not resulting from medical actions or patients' health seeking behaviours were referred as non-iatrogenic injuries. We separated iatrogenic and non-iatrogenic injuries by using both the discharge diagnosis and the external cause of injury. We further classified iatrogenic injuries as drug related and related to medical intervention (including haemorrhage or haematoma, unintentional puncture or rupture of wound, infection, complications of prosthetic device, implant and graft, or other). We also classified non-iatrogenic injuries by nature (fracture, contusion or superficial injury, internal organ injury, effect of foreign body entering orifice, dislocation, or other), region (upper extremity, head and neck, lower extremity, trunk, or other), mechanism (fall, struck by or against item, transport, nature or animal or plant, cut or piercing, poisoning, or other), place (residential areas, transportation area or street and highway, sports and athletics area, school, other institution or public administrative area, or other), and manner of intent (unintentional, intentional, assault, or undetermined). The ICD codes and external causes used for these classifications are listed in the table $\mathrm{A}$ in the appendix.

Given the study design, fatal injuries were not possible during the pre-diagnostic period so, we excluded from the analyses all fatal injuries for which a death record was identified at the time of hospital discharge.

\section{Covariates}

Information on civil status, socioeconomic status (with occupation as a proxy), and region of residence was obtained from the 1990 Swedish population and housing census. The highest educational level was retrieved from the Swedish education register. Psychiatric patients have a higher risk of injury, both in terms of self injury and unintentional injury. Because the patient register has largely complete information on inpatient care for psychiatric diseases since early 1980s and 
covers more than $80 \%$ of all hospital based outpatient specialist care since 2001, we considered that patients with cancer had pre-existing psychiatric disorders if they had any inpatient or outpatient hospital visit for psychiatric diseases since 1981 but before the pre-diagnostic period (ICD-9 codes 290-319 and ICD-10 codes F00-F99). History of inpatient care because of iatrogenic or non-iatrogenic injuries was similarly identified since 1981 but before the pre-diagnostic period for each patient with cancer.

\section{Statistical analysis}

We first calculated the incidence rates of iatrogenic and non-iatrogenic injuries during the pre-diagnostic, diagnostic, and post-diagnostic periods using number of hospital admissions divided by accumulated person months at risk. Time spent during admissions was not counted as time at risk, regardless of the discharge diagnosis. Using conditional Poisson regression, ${ }^{23}$ we subsequently estimated the incidence rate ratios and their $95 \%$ confidence intervals for injuries by comparing the incidence rates during the diagnostic and post-diagnostic periods with the incidence rates during the pre-diagnostic period in the same patients. In the analyses combining all cancers, we further split the diagnostic and post-diagnostic periods into two week intervals and calculated their corresponding incidence rate ratios. In the analyses for cancer subgroups, we calculated the incidence rate ratios per four week interval because of smaller numbers of injury events. The assumption of equal dispersion in Poisson regression was found to hold for all analyses.

We separated the analyses by sex, age at diagnosis $(\leq 54,55-64,65-74,75-84$, or $\geq 85)$, five year calendar period of diagnosis, civil status (cohabitating or not), socioeconomic status (blue collar workers, white collar workers, self employed, or others), region of residence (southern, central, or northern Sweden), education (nine years or more, less than nine years, or unknown), pre-existing psychiatric disorders (yes or no), and history of injury (yes or no). Patients with newly diagnosed psychiatric disorders after the start of pre-diagnostic period were excluded from the analysis of pre-existing psychiatric disorder $(n=42291)$.

We separately studied different subtypes of iatrogenic and non-iatrogenic injuries in the three most common cancers (prostate, breast, and colorectal cancers) and in cancers of the central nervous system, the cancer type with the largest increases in risk of both iatrogenic and non-iatrogenic injuries.

The impact of psychological distress on intentional and unintentional non-iatrogenic injuries might differ before and after a diagnosis of cancer. Distraction and reduced cognitive performance before diagnosis might lead to unintentional injuries, whereas stress related psychiatric symptoms could be more important after diagnosis, leading to increased risk of intentional injuries. We therefore calculated the incidence rate ratios during the 16 weeks before and the 16 weeks after diagnosis separately for intentional and unintentional injuries. We also calculated the incidence rate ratios for every four weeks for unintentional injury and every eight weeks for intentional injury (given smaller numbers) during the diagnostic period.

\section{Sensitivity analyses}

To assess the impact of the relation between different hospital admissions for injuries on the studied associations, we included only the first admission for injury per time period per patient in a first sensitivity analysis. To assess the specificity of the increased risks of injury around diagnosis, we conducted two similar analyses including one for patients with a diagnosis of a benign tumour during 1991-2009 according to the Swedish cancer register $(n=146099)$ and one for patients diagnosed with any diseases on the Charlson comorbidity index (excluding benign tumour and cancer) ${ }^{24}$ according to the patient register. Because the Swedish patient register has collected information on outpatient specialist visits since 2001, we included all patients with a first diagnosis of the Charlson comorbidity index diseases during 2002-09 ( $n=337294)$ and used the first hospital visit (either inpatient or outpatient) as the date of diagnosis.

To illustrate the long term risk of injuries among patients with cancer, we further calculated the standardised incidence rates of injuries among all patients from the year before diagnosis to 10 years after diagnosis, using the method of direct standardisation. The age, sex, and calendar period distribution of all person years accumulated through the entire follow-up was used as the standard population.

All analyses were performed in SAS 9.4 (SAS Institute) and Stata 13.1 (StataCorp LP). $\mathrm{P}<0.05$ was considered significant.

\section{Patient involvement}

No patients were involved in setting the research question or the outcome measures, nor were they involved in developing plans for design or implementation of the study. No patients were asked to advise on interpretation or writing up of results. There are no plans to disseminate the results of the research to study participants or the relevant patient community.

\section{Results}

\section{latrogenic injuries}

During the diagnostic period we identified 7306 iatrogenic injuries (incidence rate 0.60 per 1000 person months). Compared with the pre-diagnostic period, the increase in risk of iatrogenic injuries was larger during the diagnostic period (incidence rate ratio 7.0, 95\% confidence interval 6.6 to 7.4 ) than the post-diagnostic period (3.5, 3.3 to 3.8). Of all patients with cancer, those with cancers of the central nervous system had the largest increase in risk and those with non-melanoma skin cancer had the smallest increase, during both the diagnostic and post-diagnostic periods (table 1).

The increased risk of iatrogenic injuries started to surge from two weeks before diagnosis and peaked during two weeks after diagnosis (incidence rate ratio 48.6, 95\% confidence interval 37.3 to 63.5 ). The risk 


\begin{tabular}{|c|c|c|c|c|c|c|c|c|}
\hline & \multicolumn{2}{|c|}{ Before diagnosis } & \multicolumn{3}{|c|}{ During diagnosis } & \multicolumn{3}{|c|}{ After diagnosis } \\
\hline & $\begin{array}{l}\text { No of } \\
\text { patients }\end{array}$ & Crude IR & $\begin{array}{l}\text { No of } \\
\text { patients }\end{array}$ & Crude IR & IRR $(95 \% \mathrm{Cl})$ & $\begin{array}{l}\text { No of } \\
\text { patients }\end{array}$ & Crude IR & IRR $(95 \% \mathrm{Cl})$ \\
\hline \multicolumn{9}{|l|}{ latrogenic injury } \\
\hline All cancers & 1217 & 0.09 & 7306 & 0.60 & $7.0(6.6$ to 7.4$)$ & 2559 & 0.25 & 3.5 (3.3 to 3.8) \\
\hline Prostate & 195 & 0.08 & 1079 & 0.48 & 5.8 (5.0 to 6.8$)$ & 481 & 0.23 & 3.1 (2.6 to 3.7$)$ \\
\hline Breast & 140 & 0.07 & 978 & 0.53 & $7.5(6.2$ to 8.9$)$ & 262 & 0.15 & $2.3(1.9$ to 2.9$)$ \\
\hline Colorectal & 148 & 0.09 & 1455 & 1.05 & 11.5 (9.7 to 13.7$)$ & 453 & 0.38 & $5.0(4.0$ to 6.1$)$ \\
\hline Non-melanoma skin & 93 & 0.15 & 182 & 0.30 & $2.0(1.6$ to 2.6$)$ & 119 & 0.21 & 1.4 (1.1 to 1.9$)$ \\
\hline Lymphatic or haematopoietic & 102 & 0.11 & 320 & 0.38 & $4.0(3.2$ to 5.1$)$ & 201 & 0.29 & $3.8(2.9$ to 5.1$)$ \\
\hline Lung & 96 & 0.10 & 284 & 0.38 & $3.7(2.9$ to 4.7$)$ & 84 & 0.27 & $3.6(2.4$ to 5.5$)$ \\
\hline Central nervous system & 28 & 0.07 & 314 & 0.96 & 14.7 (10.0 to 21.6$)$ & 128 & 0.46 & $7.0(4.4$ to 11.1$)$ \\
\hline Severet & 71 & 0.11 & 335 & 0.72 & $6.6(5.1$ to 8.5$)$ & 80 & 0.57 & $6.2(3.7$ to 10.4$)$ \\
\hline Others & 372 & 0.08 & 2673 & 0.67 & $8.7(7.8$ to 9.7$)$ & 879 & 0.27 & $4.4(3.8$ to 5.1$)$ \\
\hline Smoking related cancers $†$ & 209 & 0.10 & 949 & 0.52 & $5.5(4.7$ to 6.4$)$ & 361 & 0.32 & 4.9 (3.9 to 6.2$)$ \\
\hline Alcohol related cancers & 59 & 0.11 & 351 & 0.80 & $7.6(5.8$ to 10.0$)$ & 110 & 0.43 & $4.9(3.2$ to 7.4$)$ \\
\hline \multicolumn{9}{|l|}{ Non-iatrogenic injury } \\
\hline All cancers & 5407 & 0.40 & 8331 & 0.69 & 1.9 (1.8 to 2.0$)$ & 6049 & 0.60 & $2.0(1.9$ to 2.1$)$ \\
\hline Prostate & 692 & 0.30 & 1234 & 0.55 & 2.0 (1.8 to 2.2$)$ & 1141 & 0.54 & $2.2(2.0$ to 2.4$)$ \\
\hline Breast & 652 & 0.35 & 963 & 0.52 & $1.6(1.5$ to 1.8$)$ & 930 & 0.52 & 1.8 (1.6 to 2.0$)$ \\
\hline Colorectal & 631 & 0.40 & 932 & 0.67 & $1.9(1.7$ to 2.1$)$ & 802 & 0.67 & $2.2(2.0$ to 2.5$)$ \\
\hline Non-melanoma skin & 484 & 0.78 & 567 & 0.93 & $1.2(1.1$ to 1.4$)$ & 614 & 1.10 & $1.6(1.4$ to 1.8$)$ \\
\hline Lymphatic or haematopoietic & 376 & 0.39 & 801 & 0.96 & $2.8(2.5$ to 3.2$)$ & 390 & 0.56 & 2.1 (1.8 to 2.5$)$ \\
\hline Lung & 392 & 0.43 & 692 & 0.93 & $2.5(2.2$ to 2.9$)$ & 264 & 0.84 & $3.3(2.7$ to 4.2$)$ \\
\hline Central nervous system & 129 & 0.33 & 263 & 0.81 & $2.8(2.3$ to 3.5$)$ & 140 & 0.50 & 1.8 (1.4 to 2.3$)$ \\
\hline Severet & 365 & 0.57 & 444 & 0.95 & 1.8 (1.6 to 2.1$)$ & 151 & 1.08 & 2.8 (2.1 to 3.7$)$ \\
\hline Others & 1815 & 0.41 & 2698 & 0.68 & 1.9 (1.8 to 2.0$)$ & 1757 & 0.54 & 1.9 (1.7 to 2.0$)$ \\
\hline Smoking related cancers $\dagger$ & 897 & 0.42 & 1432 & 0.78 & 2.1 (2.0 to 2.3$)$ & 763 & 0.67 & 2.6 (2.3 to 2.9$)$ \\
\hline Alcohol related cancers $†$ & 334 & 0.61 & 414 & 0.94 & 1.7 (1.5 to 2.0$)$ & 226 & 0.89 & 2.2 (1.7 to 2.7$)$ \\
\hline
\end{tabular}

decreased rapidly thereafter but remained increased throughout the diagnostic period. Increased risks were noted during the entire post-diagnostic period, with a decreasing magnitude over time (fig 1). Compared with the pre-diagnostic period, we observed a similar temporal pattern of risk increase during the diagnostic period for most cancer types (fig 2).

Compared with other patients, younger patients, cohabitating patients, patients with higher socioeconomic status or education, and patients with a diagnosis in a later calendar period had higher increases in risk of iatrogenic injuries during the diagnostic period (table 2). Patients with pre-existing psychiatric disorders or previous injury had higher incidence rates, whereas patients without pre-existing psychiatric disorders or previous injury had higher increases in risk of iatrogenic injuries. Among all patients with cancer the largest increase of risk of iatrogenic injuries was noted for infection, wound complications, and bleeding (fig 3). Similar results, although less consistent, were noted among patients with prostate, breast, colorectal, and central nervous system cancers (fig A in appendix).

\section{Non-iatrogenic injuries}

During the diagnostic period we identified 8331 non-iatrogenic injuries (incidence rate 0.69 per 1000 person months). Compared with the pre-diagnostic period there were similar increases in risk during the diagnostic (incidence rate ratio 1.9, 95\% confidence interval 1.8 to 2.0 ) and post-diagnostic (2.0, 1.9 to 2.1$)$ periods. We observed increased risks for all major cancer types, although with varying magnitude (table 1).

The risk of non-iatrogenic injuries increased dramatically from four weeks before to two weeks after diagnosis and peaked during the two weeks before diagnosis (incidence rate ratio 5.3, 95\% confidence interval 4.6 to 6.1 ). The risk increment during the post-diagnostic period declined constantly over time (fig 1). There was a similar temporal pattern for all major cancer types for the diagnostic compared with the pre-diagnostic periods (fig 4).

Older patients, patients with lower socioeconomic status or education, and patients diagnosed during earlier calendar periods presented with slightly greater increases in risk than other patients. Stronger associations were also noted for patients without pre-existing psychiatric disorders or previous injury, whereas the incidence rates were higher among patients with pre-existing psychiatric disorders or previous injury (table 2).

Except for injuries related to sports and assault, there was an increased risk for all non-iatrogenic injuries for the diagnostic compared with the pre-diagnostic period (fig 5). Given smaller numbers of events, the pattern was 

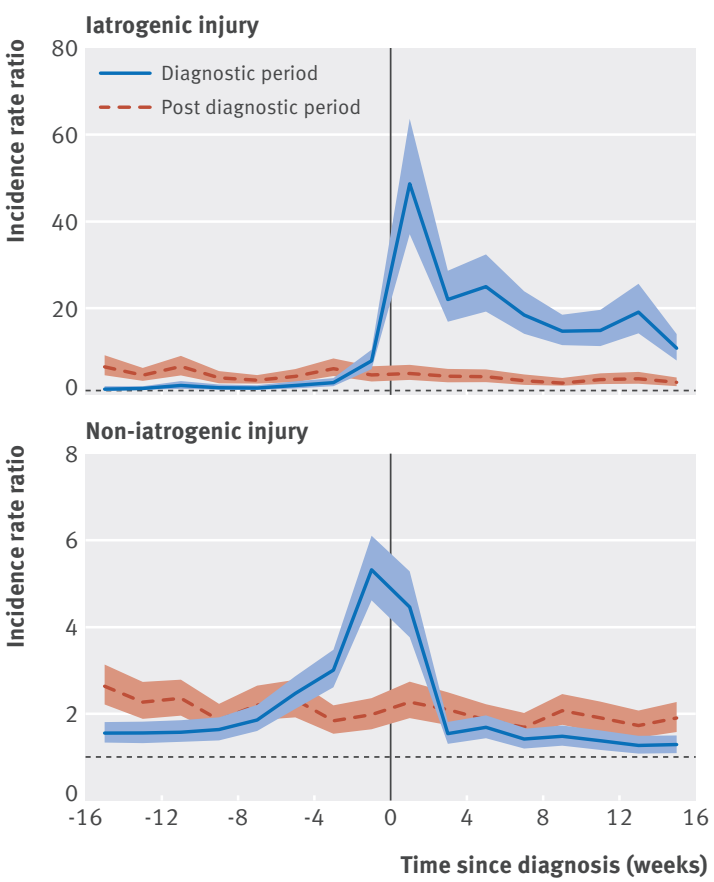

Fig 1 Injuries during diagnostic period and post-diagnostic period, compared with pre-diagnostic period, among 720901 patients with diagnosis of cancer, 1991-2009, in Sweden. Diagnostic period starts from 16 weeks before to 16 weeks after date of diagnosis. Post-diagnostic period was exact same 32 weeks one year after, whereas pre-diagnostic period was exact same 32 weeks one year before, date of diagnosis. "Weeks before and after cancer diagnosis" denotes weeks before and after one year anniversary of diagnosis for post-diagnostic period

less clear when we separately analysed patients with prostate, breast, colorectal, or central nervous system cancers (fig B in appendix).

During the 16 weeks before cancer diagnosis, the incidence rate ratio was 1.9 (95\% confidence interval 1.8 to $2.0 ; \mathrm{n}=4478$ ) for unintentional and 1.0 (0.5 to 1.9; $\mathrm{n}=20$ ) for intentional injuries, whereas during the 16 weeks after diagnosis, the figures were 2.1 (2.0 to 2.2; $\mathrm{n}=3724$ ) and 4.6 (2.0 to $10.5 ; \mathrm{n}=26$ ), respectively. There were significantly increased risks for all the four week periods before and after cancer diagnosis for unintentional injuries, but only for the eight weeks after cancer diagnosis for intentional injuries (fig $\mathrm{C}$ in appendix).

\section{Sensitivity analysis}

Results were similar when we restricted the injuries to the first hospital admission during different time periods. Compared with the pre-diagnostic period, the incidence rate ratio was 7.2 (95\% confidence interval 6.8 to 7.7) for iatrogenic and 1.9 (1.8 to 2.0) for non-iatrogenic injuries during the diagnostic period, and 3.6 (3.3 to 3.9) and 2.0 (1.9 to 2.1), respectively, during the post-diagnostic period.

For patients with benign tumours, the incidence rate ratio for iatrogenic injuries was 5.2 (95\% confidence interval 4.6 to 6.0) and for non-iatrogenic injuries 1.3 (1.2 to 1.4), whereas for patients with any Charlson comorbidity index diseases (excluding benign tumour and cancer) the incidence rate ratio was 2.8 (2.5 to 3.0) and 2.1 (2.0 to 2.2), respectively, comparing the diagnostic period with the pre-diagnostic period. Among different Charlson comorbidity index diseases, hemiplegia (incidence rate 2.81 per 1000 person months), Alzheimer's disease (2.28 per 1000 person months), and cerebrovascular diseases ( 1.43 per 1000 person months) had the highest absolute risks of non-iatrogenic injuries during the diagnostic period.

The standardised incidence rates of both iatrogenic and non-iatrogenic injuries increased rapidly from the year before diagnosis to the year after diagnosis and declined thereafter (fig 6).

\section{Discussion}

People have an increased risk of iatrogenic injuries from two weeks before a diagnosis of cancer and onward, and, importantly a highly increased risk of non-iatrogenic injuries from four weeks before until two weeks after diagnosis. Although the risks of both injury types are still clearly increased during the period after diagnosis, the excess risk is less prominent (for iatrogenic injuries) and decreases monotonically over time (for both injury types). The pronounced differences in risks of iatrogenic and non-iatrogenic injuries between diagnostic and post-diagnostic periods therefore suggest a direct impact of diagnostic procedures and primary cancer treatment on risk of injury, rather than an impact of the underlying disease alone. To the best of our knowledge, our study is the first systematic assessment of both iatrogenic and non-iatrogenic injuries shortly before and after a diagnosis of cancer.

\section{latrogenic injuries}

Iatrogenic injuries were common during the diagnostic period. Around $1 \%$ of the patients with cancer had an iatrogenic injury event requiring inpatient care during the diagnostic period, whereas only $0.2 \%$ of these patients experienced such an event during the pre-diagnostic period. The highly increased risk of iatrogenic injuries around diagnosis is not surprising because patients often undergo invasive diagnostic and therapeutic procedures and acquire other comorbidities related to the progressing malignancy and its treatment. Considering the scant data on iatrogenic injuries in medical practice in general, ${ }^{25}$ a systematic documentation of the burden of iatrogenic injuries among people with cancer-an increasingly large patient group-is clearly justified. Although an increased risk of death from medical complications after diagnosis has been reported for several common cancers, ${ }^{4-7}$ the knowledge about iatrogenic injuries during diagnostic investigation of potential cancers is limited to date. Non-melanoma skin cancer was associated with a minimal risk increase of iatrogenic injuries, whereas patients with central nervous system and colorectal cancers had the largest risk increase, probably reflecting the higher degree of complications associated with more extensive diagnostic procedures and treatment. ${ }^{26}$

Compared with other patients, those who were younger, with higher socioeconomic status and 

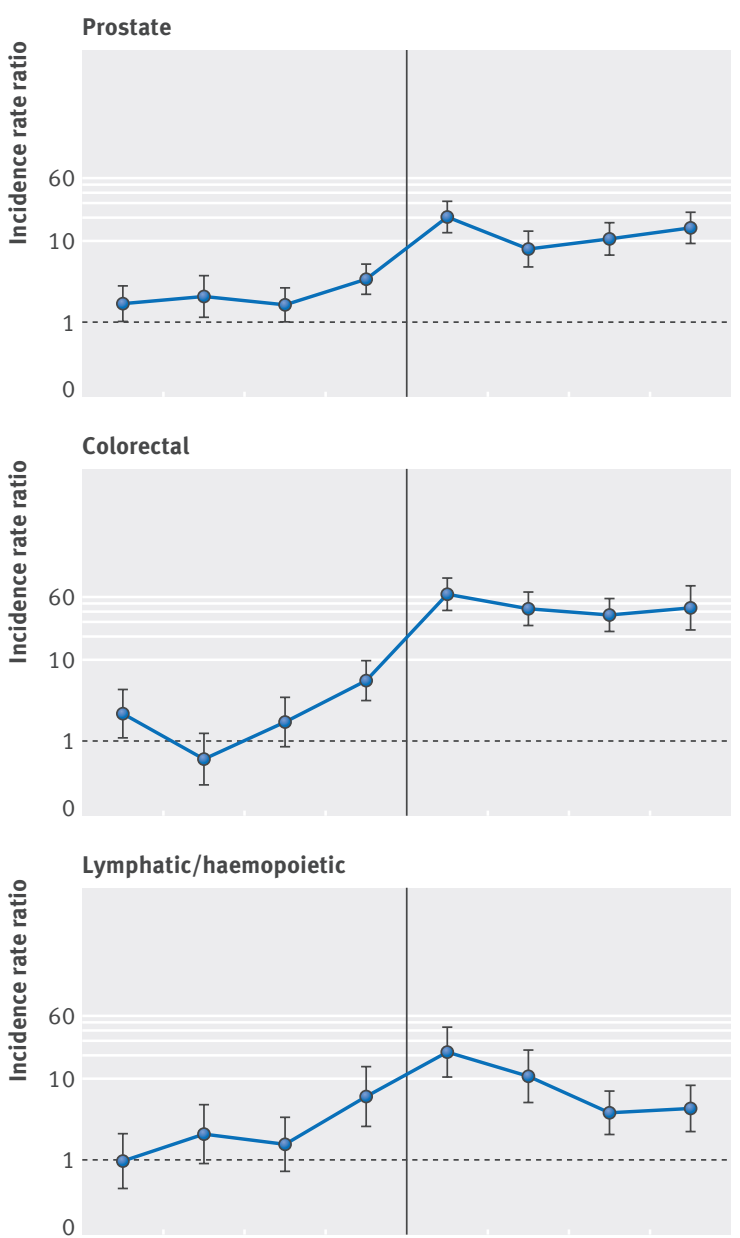

CNS

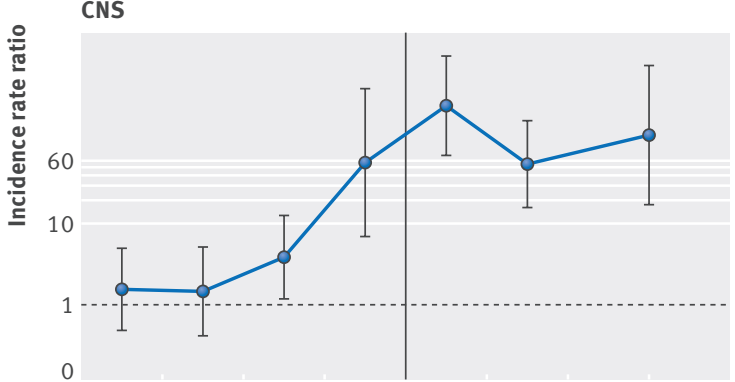

Fig 2 | latrogenic injuries during diagnostic period compared with prediagnostic period by cancer types. Diagnostic period starts from 16 weeks before to 16 weeks after date of diagnosis and prediagnostic period was exact same 32 weeks one year before date of diagnosis. For cancers of central nervous system (CNS), one incidence rate ratio for weeks 9-16 after cancer diagnosis was calculated because of small number of iatrogenic injury during these weeks of prediagnostic period
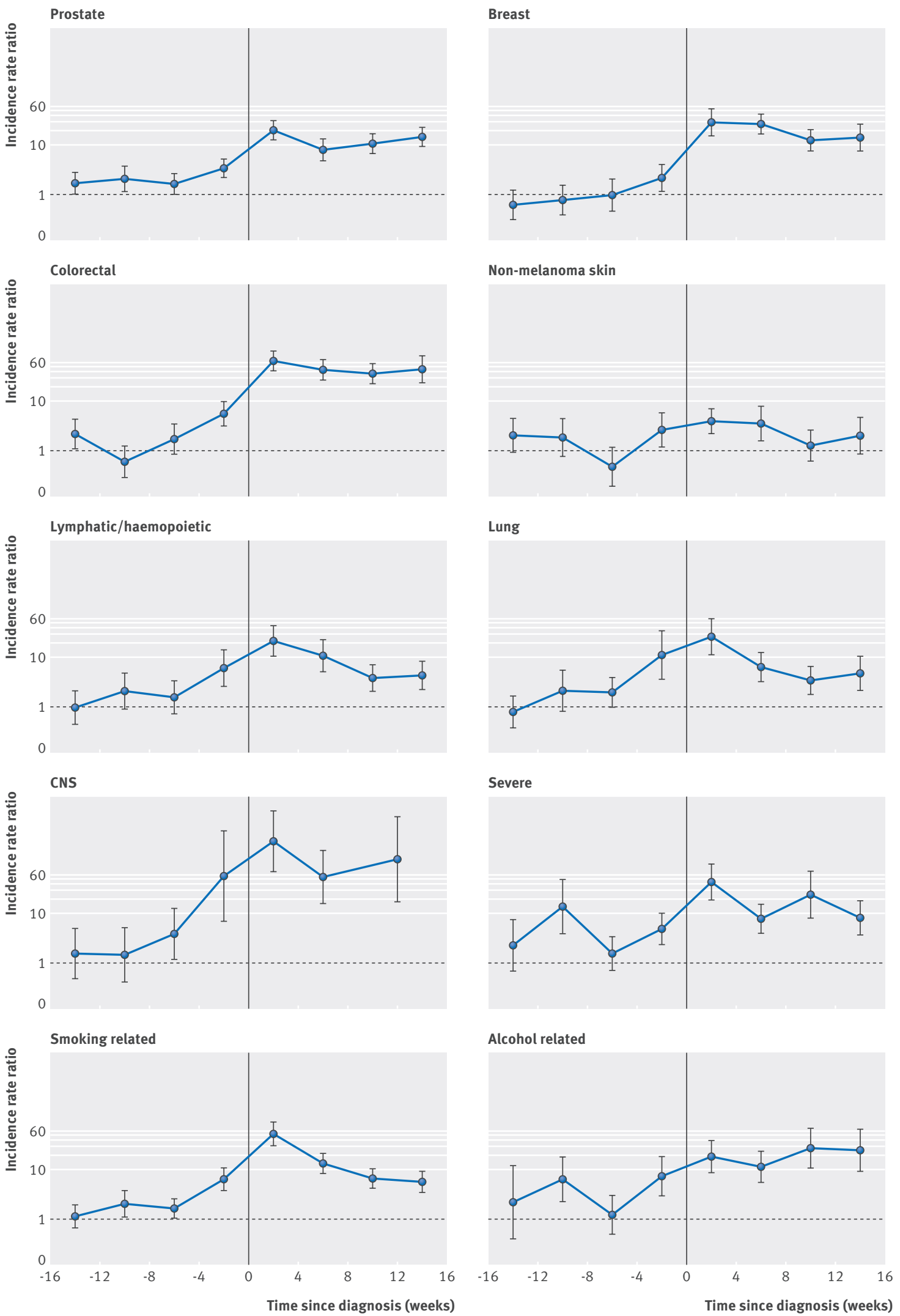

education, with a diagnosis during more recent calendar periods, and with pre-existing psychiatric disorders or previous injuries had higher risks of iatrogenic injuries during the diagnostic period, representing a high risk group of patients for potential intervention. Younger patients and patients diagnosed during more recent calendar periods might receive more aggressive treatment than older patients and those with a 
Table 2 | Incidence rates (IR) per 1000 patient months and incidence rate ratios (IRR) for injuries before and during diagnosis* in 720901 patients with diagnosis of cancer, 2001-09 in Sweden, according to demographic characteristics

\begin{tabular}{|c|c|c|c|c|c|c|c|c|c|c|}
\hline & \multicolumn{5}{|c|}{ latrogenic injury } & \multicolumn{5}{|c|}{ Non-iatrogenic injury } \\
\hline & \multicolumn{2}{|c|}{ Before diagnosis } & \multicolumn{3}{|c|}{ During diagnosis } & \multicolumn{2}{|c|}{ Before diagnosis } & \multicolumn{3}{|c|}{ During diagnosis } \\
\hline & $\begin{array}{l}\text { No of } \\
\text { patients }\end{array}$ & Crude IR & $\begin{array}{l}\text { No of } \\
\text { patients }\end{array}$ & Crude IR & IRR $(95 \% \mathrm{Cl})$ & $\begin{array}{l}\text { No of } \\
\text { patients }\end{array}$ & Crude IR & $\begin{array}{l}\text { No of } \\
\text { patients }\end{array}$ & Crude IR & $\operatorname{IRR}(95 \% \mathrm{Cl})$ \\
\hline Men & 636 & 0.09 & 3703 & 0.60 & $6.8(6.2$ to 7.4$)$ & 3183 & 0.46 & 5217 & 0.84 & $2.0(1.9$ to 2.1$)$ \\
\hline Women & 581 & 0.09 & 3603 & 0.61 & $7.3(6.6$ to 7.9$)$ & 4222 & 0.65 & 6025 & 1.03 & $1.8(1.8$ to 1.9$)$ \\
\hline \multicolumn{11}{|c|}{ Age (years) at diagnosis of cancer: } \\
\hline$\leq 54$ & 143 & 0.06 & 1441 & 0.66 & $11.3(9.5$ to 13.4$)$ & 552 & 0.23 & 686 & 0.31 & $1.4(1.2$ to 1.6$)$ \\
\hline $55-64$ & 165 & 0.06 & 1635 & 0.65 & $11.4(9.7$ to 13.4$)$ & 855 & 0.31 & 1051 & 0.42 & $1.4(1.3$ to 1.6$)$ \\
\hline $65-74$ & 347 & 0.09 & 2062 & 0.60 & $6.9(6.1$ to 7.7$)$ & 1591 & 0.42 & 2274 & 0.66 & $1.8(1.7$ to 1.9$)$ \\
\hline $75-84$ & 422 & 0.12 & 1679 & 0.56 & $4.8(4.3$ to 5.4$)$ & 2596 & 0.76 & 4399 & 1.46 & $2.2(2.0$ to 2.3$)$ \\
\hline$\geq 85$ & 140 & 0.13 & 489 & 0.53 & $4.2(3.5$ to 5.0$)$ & 1811 & 1.70 & 2832 & 3.08 & 2.1 (1.9 to 2.2$)$ \\
\hline \multicolumn{11}{|c|}{ Calendar period at cancer diagnosis: } \\
\hline $1991-95$ & 222 & 0.07 & 1187 & 0.42 & 6.8 (5.9 to 7.8$)$ & 1797 & 0.55 & 2922 & 1.03 & $2.2(2.1$ to 2.4$)$ \\
\hline $1996-2000$ & 276 & 0.08 & 1273 & 0.42 & $5.4(4.8$ to 6.2$)$ & 1948 & 0.57 & 2942 & 0.96 & $2.0(1.9$ to 2.1$)$ \\
\hline 2001-05 & 378 & 0.10 & 2186 & 0.64 & $6.6(5.9$ to 7.4$)$ & 1987 & 0.54 & 3034 & 0.89 & $1.9(1.8$ to 2.0$)$ \\
\hline 2006-09 & 341 & 0.11 & 2660 & 0.95 & 8.8 (7.9 to 9.9) & 1673 & 0.55 & 2344 & 0.84 & $1.6(1.5$ to 1.7$)$ \\
\hline \multicolumn{11}{|l|}{ Civil status: } \\
\hline Cohabitating & 629 & 0.08 & 4106 & 0.59 & 7.5 (6.9 to 8.2$)$ & 3781 & 0.46 & 5771 & 0.76 & $1.9(1.8$ to 2.0$)$ \\
\hline Non-cohabitating & 464 & 0.11 & 2339 & 0.60 & $5.9(5.4$ to 6.6$)$ & 3506 & 0.75 & 5280 & 1.27 & $2.0(1.9$ to 2.1$)$ \\
\hline \multicolumn{11}{|l|}{ Socioeconomic status: } \\
\hline White collar & 185 & 0.06 & 1832 & 0.64 & 11.2 (9.6 to 13.0$)$ & 1049 & 0.32 & 1275 & 0.41 & $1.3(1.2$ to 1.5$)$ \\
\hline Blue collar & 217 & 0.08 & 1520 & 0.64 & 7.9 (6.9 to 9.1) & 1011 & 0.34 & 1493 & 0.54 & $1.7(1.6$ to 1.9$)$ \\
\hline Self employed & 40 & 0.10 & 230 & 0.65 & $6.7(4.8$ to 9.3$)$ & 152 & 0.35 & 219 & 0.55 & $1.8(1.4$ to 2.3$)$ \\
\hline Other & 651 & 0.11 & 2863 & 0.55 & 5.3 (4.8 to 5.7$)$ & 4929 & 0.82 & 7818 & 1.48 & 2.1 (2.0 to 2.2$)$ \\
\hline \multicolumn{11}{|l|}{ Education (years): } \\
\hline$\geq 9$ & 535 & 0.08 & 3927 & 0.64 & 8.4 (7.7 to 9.2$)$ & 2655 & 0.40 & 3829 & 0.63 & $1.7(1.6$ to 1.8$)$ \\
\hline$<9$ & 577 & 0.10 & 3013 & 0.58 & 6.2 (5.6 to 6.7$)$ & 3567 & 0.61 & 5452 & 1.04 & 2.0 (1.9 to 2.1$)$ \\
\hline Unknown & 105 & 0.11 & 366 & 0.47 & $4.3(3.5$ to 5.4$)$ & 1183 & 1.29 & 1961 & 2.54 & $2.2(2.0$ to 2.4 \\
\hline \multicolumn{11}{|l|}{ Residence: } \\
\hline Southern & 222 & 0.07 & 1451 & 0.53 & 7.6 (6.6 to 8.8$)$ & 1788 & 0.56 & 2566 & 0.88 & $1.8(1.7$ to 2.0$)$ \\
\hline Central & 652 & 0.10 & 3747 & 0.63 & 6.7 (6.1 to 7.2$)$ & 4031 & 0.56 & 6209 & 0.94 & 2.0 (1.9 to 2.0$)$ \\
\hline Northern & 219 & 0.09 & 1247 & 0.58 & $6.8(5.9$ to 7.8$)$ & 1468 & 0.58 & 2276 & 1.00 & $1.9(1.8$ to 2.1$)$ \\
\hline \multicolumn{11}{|c|}{ Pre-existing psychiatric disorder: } \\
\hline Yes & 218 & 0.26 & 718 & 0.98 & $3.8(3.3$ to 4.4$)$ & 818 & 0.97 & 959 & 1.31 & $1.4(1.3$ to 1.6$)$ \\
\hline No & 886 & 0.08 & 6042 & 0.57 & 7.9 (7.4 to 8.5$)$ & 4175 & 0.36 & 6729 & 0.63 & 2.0 (1.9 to 2.1$)$ \\
\hline \multicolumn{11}{|c|}{ Previous injury requiring inpatient care: } \\
\hline Yes & 626 & 0.28 & 1748 & 0.89 & 3.3 (3.0 to 3.6) & 2458 & 1.11 & 2967 & 1.50 & $1.5(1.4$ to 1.6$)$ \\
\hline No & 591 & 0.05 & 5558 & 0.55 & 11.0 (10.1 to 11.9$)$ & 2949 & 0.26 & 5364 & 0.53 & $2.3(2.2$ to 2.4$)$ \\
\hline
\end{tabular}

*Diagnostic period starts from 16 weeks before to 16 weeks after date of diagnosis and period before diagnosis was exact same 32 weeks one year before date of diagnosis.

Injury subtypes

Drug or biological substances

Medical procedures and care

Haemorrhage or haematoma

Unintentional puncture/rupture of wound Infection

Prosthetic device, implant, graft

Unspecified, others

0.2
IRR (95\% CI)

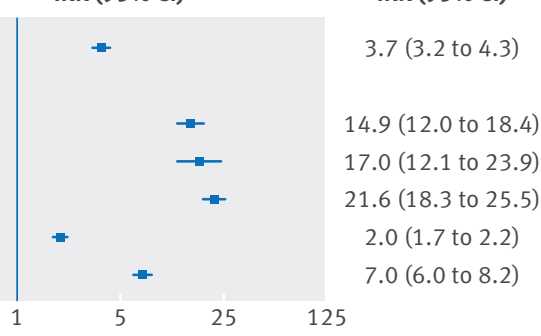

Fig 3 | Iatrogenic injuries during diagnostic period compared with pre-diagnostic period, by injury types. Diagnostic period starts from 16 weeks before to 16 weeks after date of diagnosis and pre-diagnostic period was exact same 32 weeks one year before date of diagnosis lower socioeconomic status and education might need to be strengthened. Although patients with pre-existing psychiatric diseases or injuries had a higher incidence rate of iatrogenic injuries, diagnosis of cancer apparently had a larger relative impact on iatrogenic injuries among patients without such histories.

\section{Non-iatrogenic injuries}

Patients with a new diagnosis of cancer have been shown to have a significantly increased risk of suicide and accidental death. ${ }^{9-1228}$ We extended these findings by showing that non-fatal non-iatrogenic injuries were common during the diagnostic period $(1.1 \%$ of all patients), and the risk of these injuries was already highly increased before diagnosis, especially in the four weeks before diagnosis. Date of diagnosis recorded in the Swedish cancer register represents, by definition, the date when the diagnosis is determined clinically or through morphological examinations. ${ }^{21}$ This date precedes in most cases the date when the patient is 

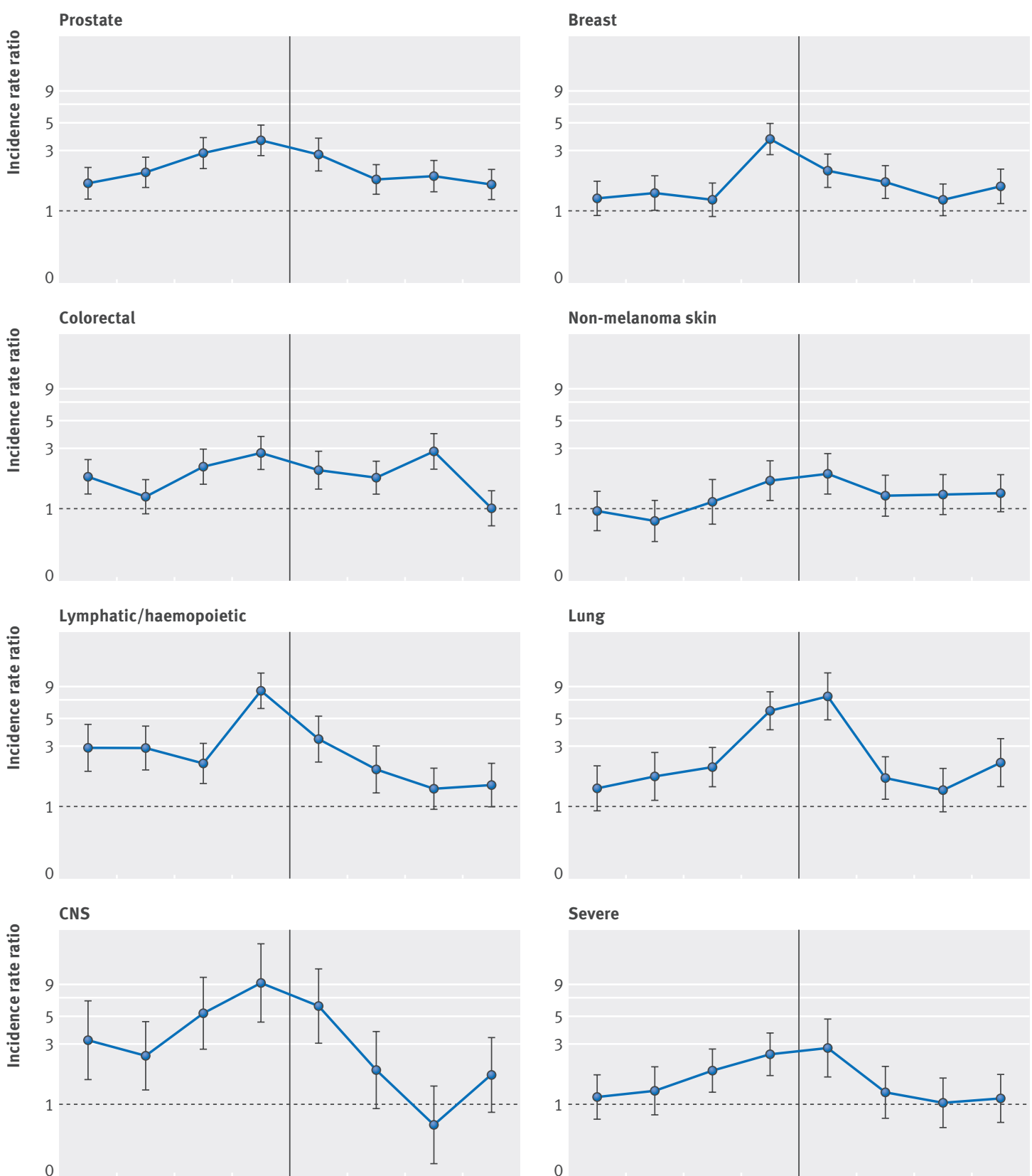

Fig 4 | Non-iatrogenic injuries during diagnostic period compared with pre-diagnostic period, by cancer types. Diagnostic period starts from 16 weeks before to 16 weeks after date of diagnosis and pre-diagnostic period was exact same 32 weeks one year before date of diagnosis
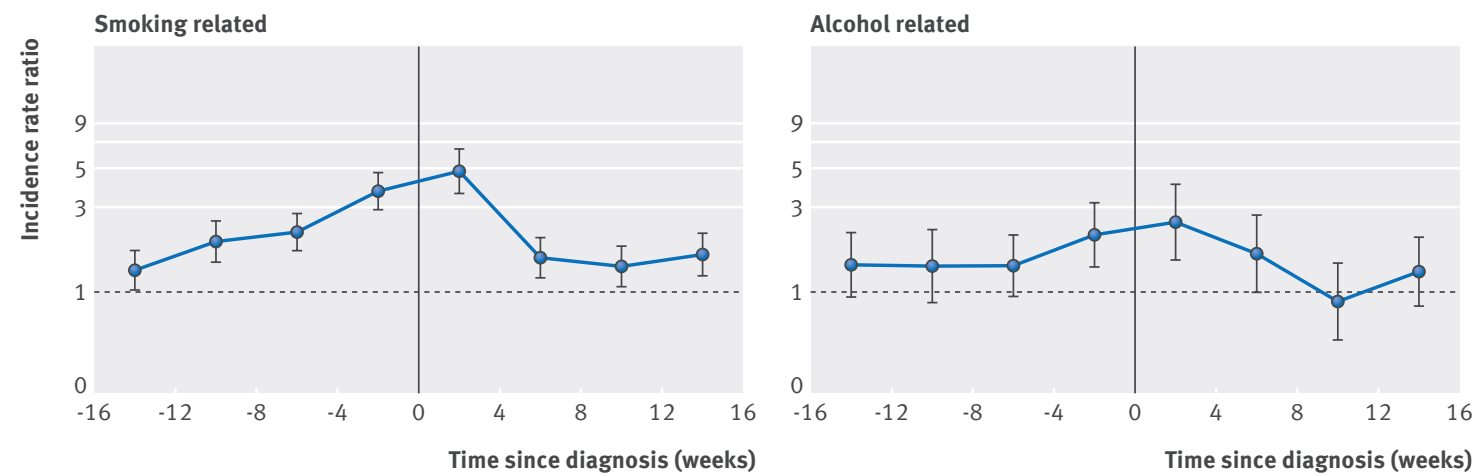

informed of the diagnosis. These findings could therefore corroborate previous studies in suggesting that the level of psychological distress is comparable, if not more severe, when patients are expecting a diagnosis of cancer than after receiving it. ${ }^{1416}$ Differences noted in the magnitude of risk increment between types of cancer also suggest a role of psychological stress as patients with non-melanoma skin cancer-a cancer with a relatively benign predicted prognosis-had the smallest increase in risk relative to patients with other cancers. 


\begin{tabular}{|c|c|c|}
\hline Injury subtypes & IRR $(95 \% \mathrm{Cl})$ & \multirow[t]{2}{*}{ IRR $(95 \% \mathrm{CI})$} \\
\hline By nature of injury & & \\
\hline Fracture & $=$ & 2.1 (2.0 to 2.1$)$ \\
\hline Contusion or superficial & 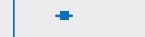 & 2.1 (1.9 to 2.4$)$ \\
\hline Open wound & $\rightarrow$ & 1.5 (1.3 to 1.8$)$ \\
\hline Internal organ injury & $=$ & 1.9 (1.7 to 2.1$)$ \\
\hline Effect of foreign body entering orifice & $\rightarrow$ & 1.8 (1.4 to 2.4$)$ \\
\hline Dislocation & 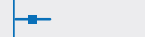 & 1.3 (1.0 to 1.7$)$ \\
\hline Others & $=$ & 1.1 (1.0 to 1.3$)$ \\
\hline \multicolumn{3}{|l|}{ By region of injury } \\
\hline Upper extremity & $=$ & 1.5 (1.4 to 1.6$)$ \\
\hline Head and neck & $=$ & 1.7 (1.6 to 1.9$)$ \\
\hline Lower extremity & $=$ & 2.1 (1.9 to 2.2$)$ \\
\hline Trunk & - & 2.3 (2.1 to 2.5$)$ \\
\hline Others & - & 1.3 (1.1 to 1.5$)$ \\
\hline \multicolumn{3}{|l|}{ By mechanism of injury } \\
\hline Fall & $=$ & 2.0 (2.0 to 2.1$)$ \\
\hline Struck by or against & $\leftarrow$ & 1.4 (1.0 to 1.8$)$ \\
\hline Transport & + & 1.1 (0.9 to 1.3$)$ \\
\hline Nature/animal/plant & $=$ & 1.1 (0.8 to 1.5$)$ \\
\hline Cut or pierce & - & $1.2(0.9$ to 1.7$)$ \\
\hline Poisoning & $\rightarrow$ & 1.4 (1.0 to 2.2$)$ \\
\hline Others & - & 1.8 (1.6 to 2.0$)$ \\
\hline \multicolumn{3}{|l|}{ By place of injury occurrence } \\
\hline Residential areas & $=$ & $2.3(2.1$ to 2.4$)$ \\
\hline Transportation area/street and highway & $=$ & 1.2 (1.0 to 1.3$)$ \\
\hline Sports and athletics area & - & $1.0(0.6$ to 1.7$)$ \\
\hline School/other institution/public administrative area & $\longrightarrow$ & 4.6 (3.0 to 7.1$)$ \\
\hline Others & $=$ & 1.9 (1.8 to 1.9$)$ \\
\hline \multicolumn{3}{|l|}{ Manner of intent } \\
\hline Unintentional (accidents) & $=$ & 1.9 (1.9 to 2.0$)$ \\
\hline Intentional self harm & $\longrightarrow$ & 1.8 (1.1 to 2.9$)$ \\
\hline Assault & - & 0.9 (0.6 to 1.3$)$ \\
\hline Undetermined and others & $\longrightarrow$ & $2.2(1.4$ to 3.6$)$ \\
\hline & 5 & \\
\hline
\end{tabular}

Fig 5 | Non-iatrogenic injuries during diagnostic period compared with pre-diagnostic period by injury types. Diagnostic period starts from 16 weeks before to 16 weeks after date of diagnosis and pre-diagnostic period was exact same 32 weeks one year before date of diagnosis
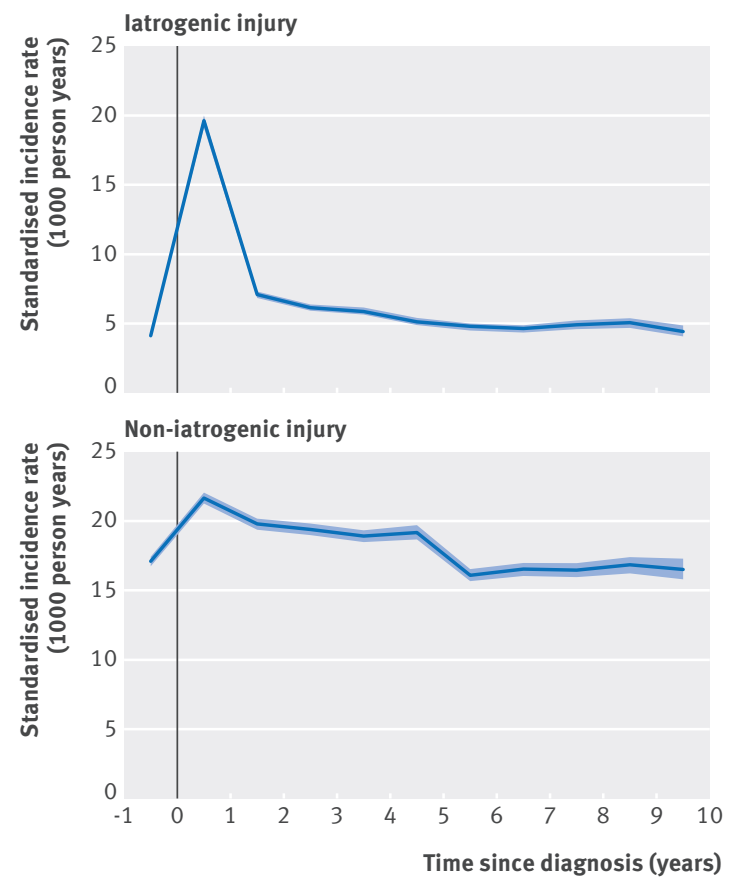

Fig 6 | Standardised incidence rates (calculated yearly and placed in middle of each year) and $95 \%$ confidence intervals of injuries from one year before cancer diagnosis to 10 years thereafter by injury types

hematopoietic, and lung cancers had both higher absolute risks and higher risk increases of non-iatrogenic injuries.

Although the highly increased risk of non-iatrogenic injuries applied to both intentional and unintentional injuries, the unintentional injuries were much more common. Furthermore, the increase in risk for unintentional injuries was similar before and after diagnosis whereas the increase in risk for intentional injuries was much more pronounced after diagnosis. The results for intentional injuries corroborates previous findings that the highest risk of suicidal behaviours occurs immediately after a diagnosis of cancer. ${ }^{12}$ Different underlying mechanisms of non-iatrogenic injuries might therefore exist before and after diagnosis. The cognitive impact of psychological distress (including impaired attention and concentration) might contribute to the increased risk of unintentional injuries before diagnosis, whereas both the cognitive impact and stress related psychiatric symptoms (including suicidal thoughts) might explain the increased risks of intentional and unintentional injuries after diagnosis. As a result, different prevention strategies should be designed to target the time periods before and after diagnosis.

\section{Specificity of findings}

The increased risk of iatrogenic injuries was substantially larger in the diagnostic period than in the post-diagnostic period and the 10 years after diagnosis, as well as in the diagnostic period for cancer compared with the diagnostic periods for benign tumours and other Charlson comorbidity index diseases. The increased risk of non-iatrogenic injuries seemed to be of similar 
magnitude for the diagnostic period for cancer compared with the post-diagnostic period and became less pronounced thereafter. The increase in risk of non-iatrogenic injuries was also greater during the diagnostic period for cancer compared with benign tumours, further suggesting the impact of severe psychological stress when people receive a life threatening diagnosis. Patients with other Charlson comorbidity index diseases had also a twofold risk of non-iatrogenic injuries during the diagnostic period; this increased risk, however, was more likely to be attributable to disease symptoms as the largest risks of non-iatrogenic injuries were noted for patients with cerebralhemisphere, Alzheimer's disease, and cerebrovascular diseases.

\section{Strengths and limitations}

Major strengths of our study include the nationwide design, large sample size, the fact that information on both the exposure and outcome was prospectively and independently collected, and the high specificity and sensitivity of the register based diagnoses of cancer and injuries, ${ }^{1819}$ substantially limiting the potential for selection and information biases. The comparison within individuals, in which each patient served as his or her own control, further eliminated potential influence of shared and unmeasured risk factors between cancer and injuries, if we assume that such risk factors are constant within individuals over a short period of time.

We included all patients in the calculation of incidence rates for injuries, whereas only patients with at least one injury during any of the study periods were included in the calculation of incidence rate ratios. As a result, the within individual comparison proxies a self controlled case series design and relies on three assumptions. ${ }^{29}$ The assumption that recurrent events must be independent should hold because we included at most one injury per month in the main analysis and results were unchanged when we restricted the analysis to the first injury during the pre-diagnostic, diagnostic, and post-diagnostic periods. The second assumption is that the occurrence of an event should not alter the probability of subsequent exposure, and violation of this assumption might lead to biased estimates of the incidence rate ratios. ${ }^{29}$ A common practice to avoid such bias is to exclude the time period leading to the exposure period from the reference period. ${ }^{29}$ By design, however, our study corrected for this possible bias by not using the time period immediately before the exposure period as the reference. The importance of the third assumption that the occurrence of event should not alter the observation period is under debate, and the bias created by violation of this assumption has been suggested as negligible. ${ }^{29}$

In the Swedish patient register, more than $90 \%$ of the injury diagnoses are reported together with the corresponding external causes, suggesting a high grade of completeness for such information. ${ }^{22}$ Although the accuracy of these external causes is yet to be validated, any potential misclassification is most likely non-differential between exposure groups and should have led the results toward null theoretically. Some of the studied iatrogenic injuries, including infections and haemorrhage, might be the reason, instead of the consequence, of the diagnostic process, partly explaining the dramatic risk increase of iatrogenic injuries before diagnosis. The fact that patients with non-melanoma skin cancer also had an increased risk of iatrogenic injuries argues against explanation by reverse causation. The potential impact of cancer symptoms, including tiredness, affected cognition, and mental status, might also affect risk of non-iatrogenic injuries and cannot be disentangled from the impact of psychological distress. This might be particularly true for patients with central nervous system cancers. ${ }^{30}$ We chose to use injuries requiring inpatient careas the main outcome because they are less prone to surveillance bias. Whether there are similarly increased risks for milder injuries during the diagnostic process warrants further study. Finally, potential roles of social support, including doctor-patient communication, on modulating the risk of injuries during diagnostic process of cancer need to be investigated in the future.

\section{Clinical implications}

Although some injuries, especially iatrogenic injuries, are hard to prevent completely given the intensive diagnostics and treatment during the diagnostic period for cancer, our findings do clearly indicate the precise and critical time window around diagnosis for the prevention of iatrogenic and non-iatrogenic injuries and the improvement of cancer care. Our study also sheds light on the high risk types of cancer and groups of patients, providing first hand evidence for clinicians and policy makers to develop targeted prevention strategies.

\section{Conclusions}

Patients with a recent diagnosis of cancer have increased risks of both iatrogenic and non-iatrogenic injuries before and after diagnosis. Our findings comprehensively show for the first time the disease burden of medical complications from diagnostic investigations for cancer. More importantly, our results call for targeted prevention of intentional and unintentional injuries, not only after diagnosis but also during the diagnostic process and primary treatment process.

\section{AUTHOR AFFILIATIONS}

${ }^{1}$ Department of Medical Epidemiology and Biostatistics, Karolinska Institutet, SE-171 77 Stockholm, Sweden

2Epidemiology and Register Centre South, Region Skåne, SE-221 85 Lund, Sweden

3Unit of Cardiovascular Disease, Institute of Environmental Medicine, Karolinska Institutet, SE-171 77 Stockholm, Sweden

${ }^{4}$ Division of Occupational and Environmental Medicine, Department of Laboratory Medicine Lund, Lund University, SE-221 00 Lund, Sweden

${ }^{5}$ Unit of Biostatistics, Institute of Environmental Medicine, Karolinska Institutet, SE-171 77 Stockholm, Sweden

${ }^{6}$ Clinical Epidemiology and Biostatistics, School of Medical Sciences, Örebro University, SE-701 82 Örebro, Sweden

${ }^{7}$ Institute of Health and Society, University of Oslo, NO-0316 Oslo, Norway

${ }^{8}$ Department of Epidemiology, Harvard T.H. Chan School of Public Health, MA-02115 Boston, USA 
${ }^{9}$ Clinical Epidemiology Unit, Department of Medicine Solna, Karolinska University Hospital, Karolinska Institutet, SE-171 77 Stockholm, Sweden

${ }^{10} \mathrm{Center}$ of Public Health Sciences, Faculty of Medicine, University of Iceland, IS-101 Reykjavík, Iceland

We thank Ruoqing Chen and Amanda Regodón Wallin for injury classification and Ninoa Malki for data management.

Contributors: DL and FF designed the study. QS performed the statistical analysis. QS, DL, and FF drafted the manuscript. All authors analysed and interpreted the data and approved the final version. QS and DL contributed equally to the work, had full access to all the data in the study, and take responsibility for the integrity of the data and the accuracy of the data analysis. FF is guarantor.

Funding: This work was supported by the Swedish Cancer Society (grant No CAN 2014/417), the Swedish Research Council for Health, Working Life and Welfare (grant No 2012-0498), and the China Scholarship Council. FF received a Swedish Society for Medical Research and Karolinska Institute research associate award. H-OA (Dnr: 2368/10-221) and SC (Dnr: 2368/10-221) received Karolinska Institute distinguished professor awards.

Competing interest: All authors have completed the Unified Competing Interest form at www.icmje.org/coi_disclosure.pdf (available on request from the corresponding author) and declare that: no support from any company for the submitted work; no relationships with any company that might have an interest in the submitted work in the previous three years; no financial relationships with any organizations that may be relevant to the submitted work; and no other relationships or activities that may influence the submitted work.

Ethical approval: The study was approved by the ethical review board in Stockholm, Sweden.

Data sharing: No additional data available.

Transparency declaration: The lead author affirms that the manuscript is an honest, accurate, and transparent account of the study being reported; that no important aspects of the study have been omitted; and that any discrepancies from the study as planned have been explained.

This is an Open Access article distributed in accordance with the Creative Commons Attribution Non Commercial (CC BY-NC 3.0) license, which permits others to distribute, remix, adapt, build upon this work non-commercially, and license their derivative works on different terms, provided the original work is properly cited and the use is noncommercial. See: http://creativecommons.org/licenses/by-nc/3.0/.

1 Baade PD, Fritschi L, Eakin EG. Non-cancer mortality among people diagnosed with cancer (Australia). Cancer Causes Control 2006:17:287-97. doi:10.1007/s10552-005-0530-0.

2 Weingart SN, Wilson RM, Gibberd RW, Harrison B. Epidemiology of medical error. BMJ 2000;320:774-7. doi:10.1136/bmj.320.7237.774.

3 Camidge DR, Stockton DL, Frame S, Wood R, Bain M, Bateman DN. Hospital admissions and deaths relating to deliberate self-harm and accidents within 5 years of a cancer diagnosis: a national study in Scotland, UK. BrJ Cancer 2007;96:752-7. doi:10.1038/sj.bjc.6603617.

4 Iversen LH, Bülow S, Christensen IJ, Laurberg S, Harling H. Danish Colorectal Cancer Group. Postoperative medical complications are the main cause of early death after emergency surgery for colonic cancer. Br/ Surg 2008:95:1012-9. doi:10.1002/bjs.6114.

5 Riihimäki M, Thomsen H, Brandt A, Sundquist J, Hemminki K. What do prostate cancer patients die of?Oncologist 2011;16:175-81. doi:10.1634/theoncologist.2010-0338

6 Riihimäki M, Thomsen H, Brandt A, Sundquist J, Hemminki K. Death causes in breast cancer patients. Ann Oncol 2012;23:604-10. doi:10.1093/annonc/mdr160

7 Riihimäki M, Thomsen H, Sundquist K, Hemminki K. Colorectal Cancer Patients: What Do They Die Of?Frontline Gastroenterol 2012;3:1439doi:10.1136/flgastro-2012-100141

8 Anguiano L, Mayer DK, Piven ML, Rosenstein D. A literature review of suicide in cancer patients. Cancer Nurs 2012;35:E14-26. doi:10.1097/ NCC.0b013e31822fc76c.
9 Lu D, Fall K, Sparén P, et al. Suicide and suicide attempt after a cancer diagnosis among young individuals. Ann Oncol 2013;24:3112-7. doi:10.1093/annonc/mdt415.

10 Yamauchi T, Inagaki M, Yonemoto N, et al. JPHC Study Group. Death by suicide and other externally caused injuries following a cancer diagnosis: the Japan Public Health Center-based Prospective Study. Psychooncology 2014;23:1034-41. doi:10.1002/pon.3529.

11 Dalela D, Krishna N, Okwara J, et al. Suicide and accidental deaths among patients with non-metastatic prostate cancer. BJU Int 2016:118:286-97. doi:10.1111/bju.13257.

12 Fang F, Fall K, Mittleman MA, et al. Suicide and cardiovascular death after a cancer diagnosis. N Engl/ Med 2012;366:1310-8. doi:10.1056/ NEJMoa1110307.

13 Merrill RM, Brown RJ, Alder S, et al. Psychological disorders among children and the parents of children undergoing cancer workup. J Psychosoc Oncol 2007;25:1-18. doi:10.1300/J077v25n03_01.

14 Awsare NS, Green JSA, Aldwinckle B, Hanbury DC, Boustead GB, McNicholas TA. The measurement of psychological distress in men being investigated for the presence of prostate cancer. Prostate Cancer Prostatic Dis 2008:11:384-9. doi-10.1038/pcan.2008.21

15 Brasso K, Ladelund S, Frederiksen BL, Jørgensen T. Psychological distress following fecal occult blood test in colorectal cancer screening--a population-based study. Scand / Gastroenterol 2010:45:1211-6. doi:10.3109/00365521.2010.485355.

16 Gøtzsche PC, Jørgensen KJ. Screening for breast cancer with mammography. Cochrane Database Syst Rev 2013;6:CD001877. surveillance for prostate cancer affect quality of life? A systematic review. Eur Urol 2015;67:637-45. doi:10.1016/j.eururo.2014.10.028.

18 Barlow L, Westergren K, Holmberg L, Talbäck M. The completeness of the Swedish Cancer Register: a sample survey for year 1998. Acto Oncol 2009;48:27-33. doi:10.1080/02841860802247664.

19 Kassel JD, Stroud LR, Paronis CA. Smoking, stress, and negative affect: correlation, causation, and context across stages of smoking. Psychol Bull 2003;129:270-304. doi:10.1037/0033-2909.129.2.270.

20 Herman JP. Neural pathways of stress integration: relevance to alcohol abuse. Alcohol Res 2012;34:441-7.

21 Socialstyrelsen. Väntetider i cancervården-från remiss till diagnos och behandling. National Board of Health and Welfare, 2012. http://www. socialstyrelsen.se/publikationer2012/2012-10-28.

22 Ludvigsson JF, Andersson E, Ekbom A, et al. External review and validation of the Swedish national inpatient register. BMC Public Health 2011;11:450. doi:10.1186/1471-2458-11-450.

23 Armstrong BG, Gasparrini A, Tobias A Conditional Poisson models: a flexible alternative to conditional logistic case cross-over analysis. BMC Med Res Methodol 2014;14:122 doi:10.1186/1471-2288-14-122.

24 Quan H, Li B, Couris CM, et al. Updating and validating the Charlson comorbidity index and score for risk adjustment in hospital discharge abstracts using data from 6 countries. Am J Epidemiol 2011;173:67682. doi:10.1093/aje/kwq433.

25 Makary MA, Daniel M. Medical error-the third leading cause of death in the US. BMJ 2016;353:i2139. doi:10.1136/bmj.i2139.

26 Hoang-Xuan K, Camilleri-Broët S, Soussain C. Recent advances in primary CNS lymphoma. Curr Opin Oncol 2004:16:601-6. doi:10.1097/01.cco.0000142927.88773.7e.

27 Goodwin JS, Hunt WC, Samet JM. Determinants of cancer therapy in elderly patients. Cancer 1993;72:594-601. doi:10.1002/1097-0142(19930715)72:2<594:AIDCNCR2820720243>3.0.CO;2-\#.

28 Urban D, Rao A, Bressel M, Neiger D, Solomon B, Mileshkin L. Suicide in lung cancer: who is at risk?Chest 2013;144:1245-52. doi:10.1378/ chest.12-2986.

29 Whitaker HJ, Hocine MN, Farrington CP. The methodology of self-controlled case series studies. Stat Methods Med Res 2009;18:7-26. doi:10.1177/0962280208092342.

30 Heimans JJ, Taphoorn MJ. Impact of brain tumour treatment on quality of life. / Neurol 2002;249:955-60. doi:10.1007/s00415-002-0839-5.

Appendix: Supplementary material (ICD codes, table A, figs A-C) 